\title{
Assessment of Carotid Plaque Vulnerability Using Structural and Geometrical Determinants
}

\author{
Zhi-Yong Li, PhD*,**; Tjun Tang, MRCS*; Jean U-King-Im, FRCR*; Martin Graves, MSc*; \\ Michael Sutcliffe, PhD**; Jonathan H Gillard, MD, FRCR*
}

\begin{abstract}
Background Because many acute cerebral ischemic events are caused by rupture of vulnerable carotid atheroma and subsequent thrombosis, the present study used both idealized and patient-specific carotid atheromatous plaque models to evaluate the effect of structural determinants on stress distributions within plaque.

Methods and Results Using a finite element method, structural analysis was performed using models derived from in vivo high-resolution magnetic resonance imaging (MRI) of carotid atheroma in 40 non-consecutive patients (20 symptomatic, 20 asymptomatic). Plaque components were modeled as hyper-elastic materials. The effects of varying fibrous cap thickness, lipid core size and lumen curvature on plaque stress distributions were examined. Lumen curvature and fibrous cap thickness were found to be major determinants of plaque stress. The size of the lipid core did not alter plaque stress significantly when the fibrous cap was relatively thick. The correlation between plaque stress and lumen curvature was significant for both symptomatic $(\mathrm{p}=0.01$; correlation coefficient: 0.689 ) and asymptomatic patients ( $\mathrm{p}=0.01$; correlation coefficient: 0.862 ). Lumen curvature in plaques of symptomatic patients was significantly larger than those of asymptomatic patients $\left(1.50 \pm 1.0 \mathrm{~mm}^{-1} \mathrm{vs}\right.$ $\left.1.25 \pm 0.75 \mathrm{~mm}^{-1} ; \mathrm{p}=0.01\right)$.

Conclusion Specific plaque morphology (large lumen curvature and thin fibrous cap) is closely related to plaque vulnerability. Structural analysis using high-resolution MRI of carotid atheroma may help in detecting vulnerable atheromatous plaque and aid the risk stratification of patients with carotid disease. (Circ J 2008; 72:
\end{abstract} 1092-1099)

Key Words: Atherosclerosis; Carotid artery; MRI; Plaque vulnerability; Structural analysis

$\mathbf{R}$ upture of atheromatous plaque in the carotid artery often leads to thrombosis and subsequent stroke! This is potentially preventable by carotid revascularization techniques, and the degree of luminal stenosis is commonly used in clinical practice as an indicator for carotid endarterectomy or carotid artery angioplasty/stenting. Carotid endarterectomy has been shown to be beneficial in patients with symptomatic high-grade (70-99\%) stenosis, 2,3 but it is more difficult to draw conclusions about possible benefits for patients with moderate carotid stenosis. Moreover, the debate continues about whether or not asymptomatic patients with a moderate stenosis should undergo endarterectomy, despite the findings of the recent Asymptomatic Carotid Surgery Trial (ACST)4 Previous studies have shown that vulnerable plaque (ie, prone to rupture with thromboembolic complications) has a thin fibrous cap, a large lipid core and a high inflammatory cell burden,5 Therefore, investigating plaque structure and morphology may aid in the risk stratification of patients with a moderate luminal stenosis.

Although the original concept of vulnerable plaque was derived from the coronary circulation, there are compelling

(Received October 14, 2007; revised manuscript received January 18, 2008; accepted February 6, 2008)

*University Department of Radiology, Addenbrooke's Hospital, **Department of Engineering, University of Cambridge, Cambridge, UK

Mailing address: Zhi-Yong Li, PhD, University Department of Radiology, Box 219, Level 5, Cambridge University Hospitals Foundation Trust, Cambridge CB2 2QQ, UK. E-mail: ZYL22@ cam.ac.uk All rights are reserved to the Japanese Circulation Society. For permissions, please e-mail: cj@j-circ.or.jp reasons why it also applies to the carotid circulation?-12 The mechanism of plaque rupture is not entirely clear, but is thought to be a multifactorial process involving thinning and weakening of the fibrous cap by enzymes secreted by activated macrophages, and biomechanical stress as the trigger leading to plaque rupture. ${ }^{13-15}$ From the point of view of structural analysis, plaque rupture is structural failure when the plaque cannot resist the hemodynamic blood pressure and shear stress exerted on it. In the process of plaque rupture, an excessive concentration of stress at a weak site on the plaque surface is considered to be an important factor ${ }^{16-18}$

The mechanism of plaque rupture has been widely studied using computational simulations ${ }^{17,19,20}$ Hung et al used histology-based 2-dimensional (D) solid models for arterial plaque and found that a thin fibrous cap and a large lipid core are important determinants of increased plaque stress ${ }^{21}$ Cheng et al used a finite element model based on histology to analyze coronary lesions, and their data suggested that the concentration of circumferential tensile stress in the lesion may play an important role in plaque rupture and myocardial infarction ${ }^{17}$ Tang et al used an ex vivo magnetic resonance imaging (MRI)-based flow-structure interaction model to study the interaction between flow and plaque, and suggested that large cyclic stress-strain variations in the plaque under pulsatile flow pressure may lead to plaque fatigue and possible rupture?2 Imoto et al used longitudinal structural analysis of plaque rupture and revealed that plaque shape, size and remodeling may be associated with plaque rupture ${ }^{23}$ We previously used a blood flow and plaque interaction model to demonstrate 
that fibrous cap thickness is critical to plaque stability 24

In the present study, we used a finite element method (FEA) and performed structural analysis of idealized plaque models to examine the determinants of plaque vulnerability by varying the fibrous cap thickness, lipid core size and lumen curvature. Structural analyses were also performed based on geometry derived from in vivo high-resolution MRI. The stress distributions within atherosclerotic carotid plaques were calculated and the maximum stresses were used to evaluate vulnerability.

\section{Methods}

\section{Idealized Plaque Models}

Various idealized plaque cross-section models were designed to examine the effect of different geometries on the stress distribution within plaque. The idealized model consisted of a generalized cross-section of an artery with an atheromatous plaque, a circular lumen and a blunt, crescent-shaped lipid core (Fig 1). In this model, the lumen and vessel diameters were chosen to be $3 \mathrm{~mm}$ and $10 \mathrm{~mm}$, respectively (corresponding to $70 \%$ luminal stenosis), and the vessel wall thickness was $1.5 \mathrm{~mm}^{25,26}$ The fibrous cap thickness $(d)$ and lipid core size (A) were varied to study their effect on stress distribution within the plaque.

Several typical lumen shapes based on the baseline model were investigated by keeping luminal diameter, fibrous cap thickness and lipid area constant. The luminal surface area was kept constant to ensure equal pressure load at the same blood pressure.

\section{Multi-Spectral In Vivo MRI-Based Models}

In vivo multi-sequence MRI data were acquired from 40 non-consecutive individuals (20 symptomatic, 20 asymptomatic; 29 males, median age 71.5 years (range 52-83)), recruited from a specialist neurovascular clinic. Informed written consent was given by all patients and the study was approved by the Internal Review Board. The median time from symptoms to carotid endarterectomy in the symptomatic cohort was 7 weeks (range 2-12). All symptomatic individuals had recently experienced either a retinal or cortical transient ischemic attack or a complete hemispheric stroke within the previous 6 months in the designated territory. Asymptomatic patients had experienced no symptoms within the 6 months before MRI.

Imaging studies were conducted using a 1.5-Tesla whole body system (GE Medical Systems, Milwaukee, WI, USA) with a custom-designed 4-channel phased array surface coil (PACC, Machnet BV, Elde, The Netherlands) wrapped around the neck and secured by a soft cervical collar. Additionally, patients were placed in a foam headrest to minimize motion artifacts. After an initial coronal localizer study, an axial 3-D Time-of-Flight (TOF) MR angiography was performed to identify the carotid bifurcation and the region of maximal stenosis. Axial images were acquired through the common carotid artery, $6 \mathrm{~mm}$ ( 2 slices) below the carotid bifurcation to a point $6 \mathrm{~mm}$ ( 2 slices) distal to the extent of the stenosis identified on the TOF sequence. This method ensured that the entire plaque was imaged and also facilitated image co-registration. The following 2-D, ECGgated, blood-suppressed, fast spin echo pulse sequences were used: intermediate $\mathrm{T}_{2}$ weighted with fat saturation, $\mathrm{T}_{2}$ weighted, short $\mathrm{T}_{1}$ inversion-recovery (STIR), and $\mathrm{T}_{1}$ weighted. The voxel size was $0.39 \times 0.39 \times 3 \mathrm{~mm}$ in all cases. The field of view was $10 \mathrm{~cm}$ and matrix size $256 \times 256$.

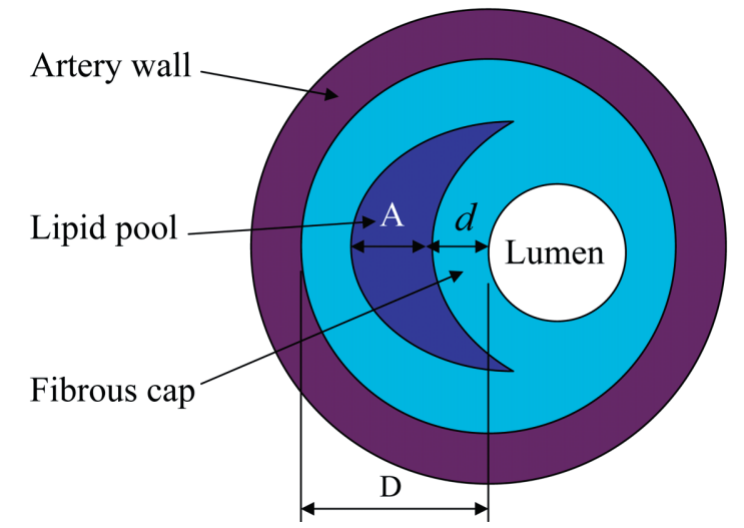

Fig 1. Model of an atheromatous plaque ( $d$ : fibrous cap thickness; A: lipid core size; D: the distance between lumen to vessel wall (the larger side)).

Slices were acquired sequentially with a 3-mm thickness and no inter-slice gap.

The conduct of the MRI studies did not cause a delay in any surgical intervention. Plaque geometry was obtained from the multi-spectral images. The regions of interest (ROI) were delineated manually with MR segmentation software (CMR tools v4.0, Imperial College, London, UK), corresponding to the plaque constituents of lipid core, fibrous cap, vessel wall and lumen. These components have different signal characteristics when imaged using our multi- sequence protocol. For example, the fibrous cap is particularly bright on STIR imaging, and the lipid core is dark on intermediate $\mathrm{T} 2$ weighted imaging with fat suppression. These techniques have been validated in a previous study against the histological gold standard26,27

Once delineated, control vertices were exported from the segmentation software and imported into a specialist engineering package (MSC. Patran 2004 r2, MSC Software Corp, Santa Ana, CA, USA and ABAQUS Software V6.5, Rhodes Island, Providence, RI, USA) where the contours were reconstructed using a closed B-spline technique to form the geometry for mesh generation.

\section{Structural Analysis}

Structural analysis was performed using both the idealized plaque and MRI-based models. A standardized mean pressure was uniformly applied to the internal vessel wall in each numerical simulation. The pressure was assumed negligible on the outside vessel surface and the plaque was considered stress-free at zero pressure (no residual stress).

Plaque components were assumed to be hyper-elastic materials. In order to model this hyper-elastic behavior, a 2-term Ogden strain energy formulation was chosen to simulate the hyper-elastic stress/strain relation for the mechanical properties of plaque components 28 This model was originally developed to describe large deformation isotropic elasticity in incompressible elastomeric solids ${ }^{28}$ It can be described in the following form:

$W=\sum_{i=1}^{N} \frac{\mu_{i}}{\alpha_{i}} J^{-\frac{\alpha_{i}}{3}}\left(\lambda_{1}^{a_{i}}+\lambda_{2}^{a_{i}}+\lambda_{3}^{a_{i}}-3\right)+4.5\left(K^{-\frac{1}{3}}-1\right)^{2}$

where $N$ is the number of terms and was taken to be 2 in this simulation. $\lambda_{i}$ are the principle stretch ratios, $J$ is the Jacobian modulus, and $K$ is the bulk modulus. $\mu$ iare moduli 


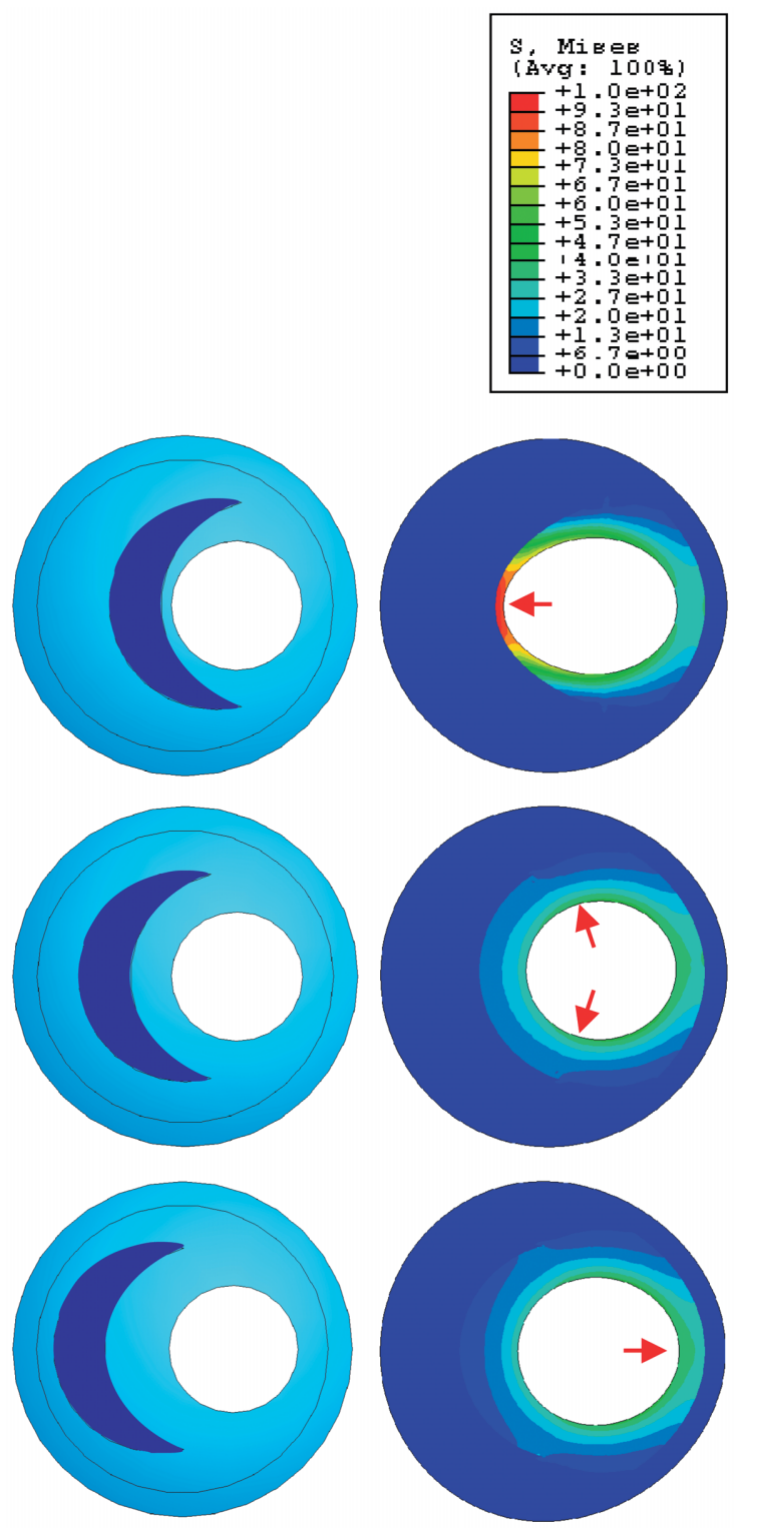

Fig 2. Effect of fibrous cap thickness on von Mises plaque stress distribution. Stress distributions within 3 different plaques with increasing fibrous cap thickness (from top to bottom) are shown. The color scale shows the absolute stress values within the plaque, with the red arrow pointing to the maximum stresses. It is interesting that in the bottom plaque (with a thick fibrous cap), the maximum stress was on the contralateral side of the plaque away from the fibrous cap, but it should be noted that the maximum stress acting there was still much lower than in the top plaque (with a thin fibrous cap).

constants and $\mu_{1}=-\mu_{2} . \alpha_{i}$ are exponent constants and $\alpha_{1}=$ $-a_{2}$. The values of the carotid plaque components were chosen to be the same as in our previous work. ${ }^{27}$

The computational structural analysis was performed using a FEA. The complex structure of a plaque model was first divided into smaller subunits designated as elements. The total number of elements for each model was approximately 20,000. The structure was meshed with quadrilateral plan strain elements. The equivalent stress for each element was calculated. An internal pressure of $15 \mathrm{kPa}(115 \mathrm{mmHg})$ was applied to the lumen wall, representing a mean physiological systolic blood pressure in the carotid artery. The numerical simulation was carried out using an FEA package

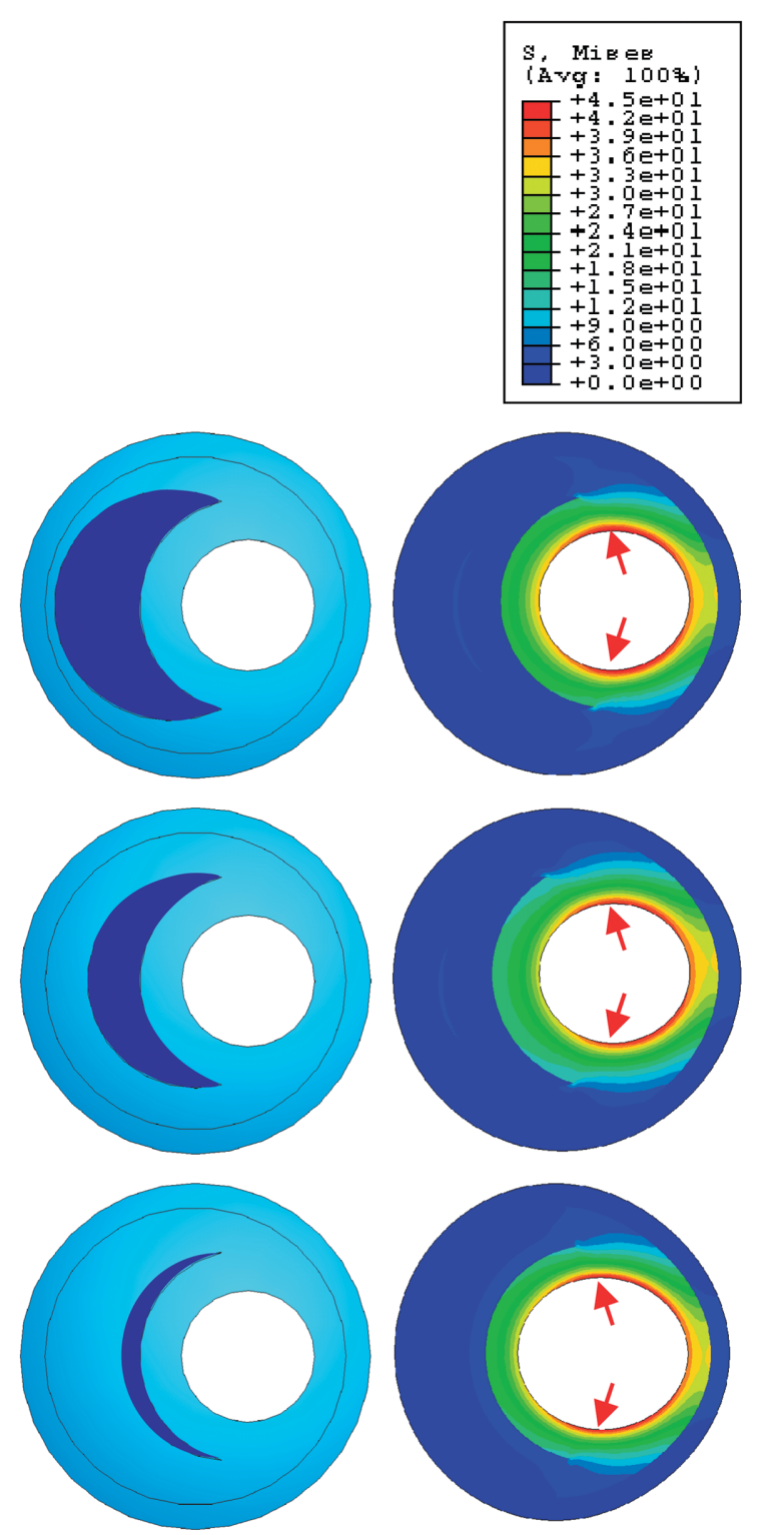

Fig 3. Effect of lipid core size on plaque stress distribution. Stress distributions within 3 different plaques with decreasing lipid core size (from top to bottom: 2A, A, 0.5A) are shown. The color scale shows the absolute stress values within the plaque, with the red arrow pointing to the maximum stresses.

ABAQUS (ABAQUS V6.5). Contour plots of the von Mises stresses were displayed using ABAQUS post processing. Von Mises stress is used to estimate yield criteria for ductile materials. It is calculated by combining stresses in 2 or 3 dimensions. Stress is in general a complicated 6-D tensor quantity. Von Mises stress reduces this to a single number (a scalar) for the purposes of calculating yield criteria.

The study analyzed the stress distributions within several idealized plaque models when varying the structural characteristics, followed by 40 patient-specific plaque models.

\section{Results}

Color-coded stress contours were used to represent the values of the von Mises stresses. The differences in stress 
A
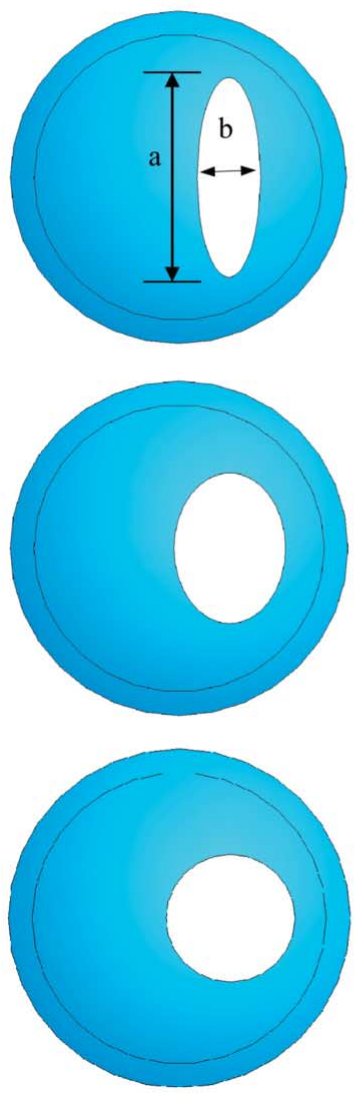
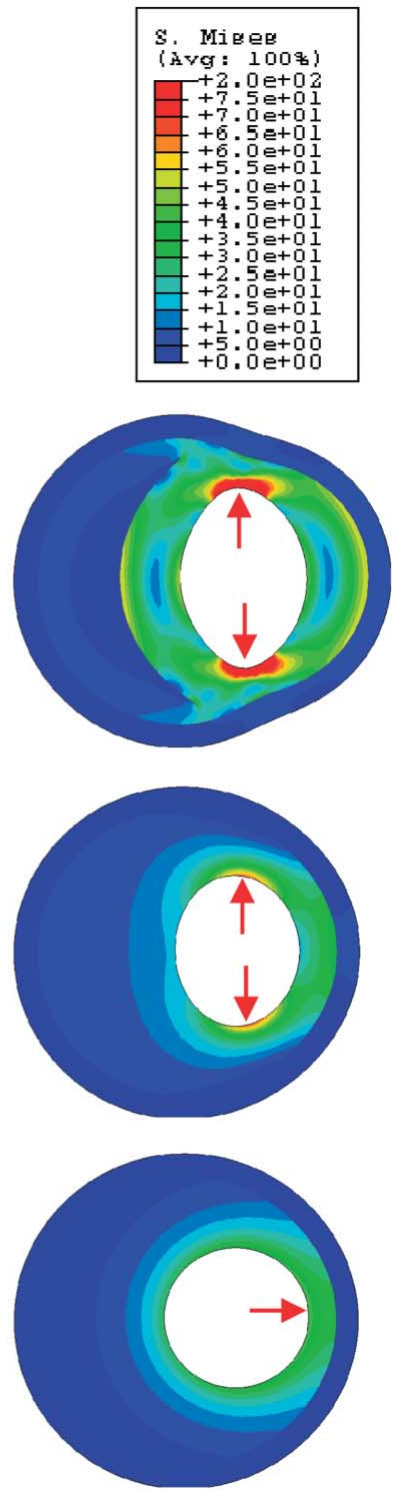

B
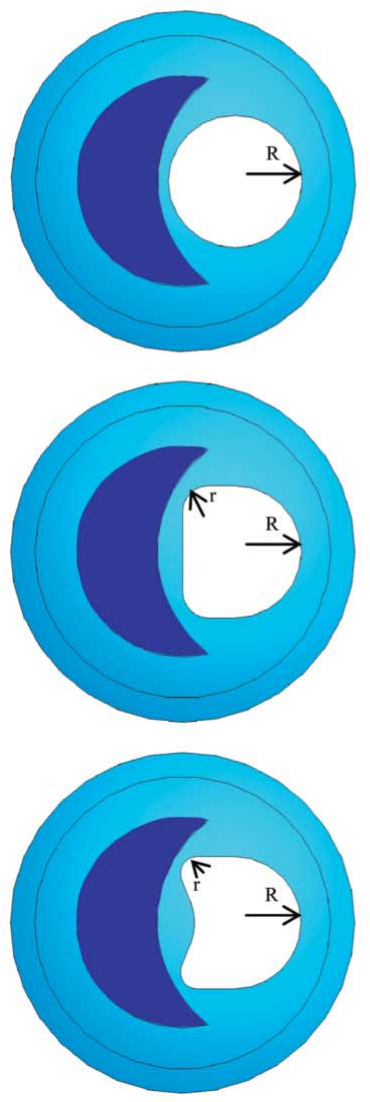
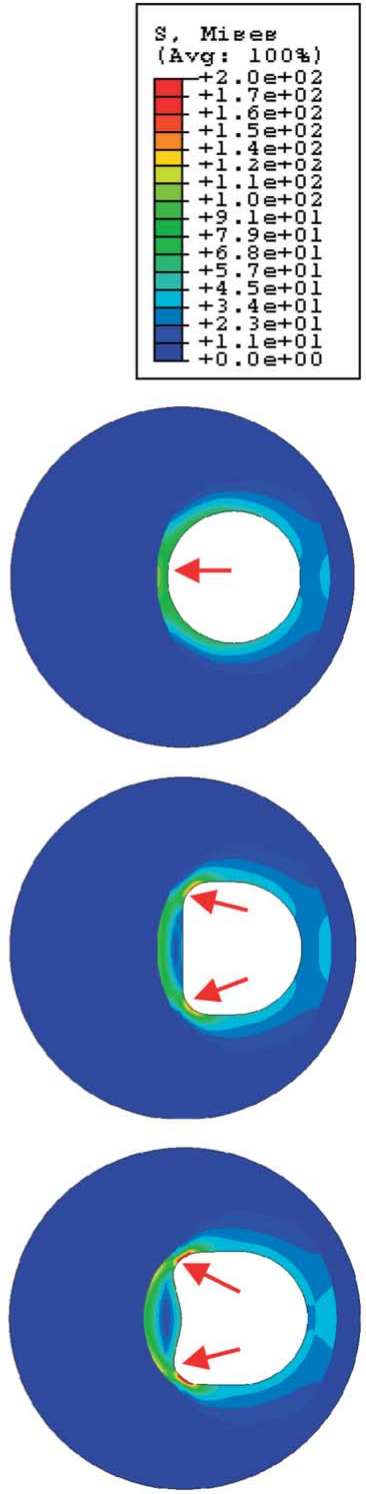

Fig 4. Effect of lumen curvature on plaque stress distribution. Stress distributions within 3 plaques with different lumen shapes are shown. The color scale shows the absolute stress values with the red arrow pointing to the maximum stresses. (A) Lumen varying from a circle to an ellipse (from top to bottom: $a / b=3,1.5,1$ ). (B) Lumen shape distorted in progressive steps: circular $(\mathrm{r} / \mathrm{R}=1)$, flattened $(\mathrm{r} / \mathrm{R}=0.55)$, and crescent $(\mathrm{r} / \mathrm{R}=0.38)$. The lumen surface area was kept constant, ensuring equal pressure load at equal blood pressure.

distributions when varying the thickness of the fibrous cap are shown in Fig 2. Keeping the size of the lipid core constant $(\mathrm{A}=0.3 \mathrm{D})$, there was an increase in maximum stress when the fibrous cap thickness decreased. Stress concentrations can be found at the thinnest part of the fibrous cap (Fig 2). In contrast, when the fibrous cap was relatively thick, there was only a slight increase in plaque stress when the lipid core increased while keeping the fibrous cap thickness constant (Fig 3).

With regards to the effect of lumen shape and curvature on plaque stress distribution, 2 types of plaque models were used. When the lumen was a circle, the maximum stress was $42.42 \mathrm{kPa}$. When the lumen was an ellipse, plaque stress increased to $65.49 \mathrm{kPa}$ (when $\mathrm{a} / \mathrm{b}=1.5$ ) and $164.9 \mathrm{kPa}$ (when $a / b=3$ ). We also studied the effect of lumen curvature when the lumen shape was distorted in progressive steps: circular $(\mathrm{r} / \mathrm{R}=1)$, flattened $(\mathrm{r} / \mathrm{R}=0.55)$, and crescent $(\mathrm{r} / \mathrm{R}=0.38)$.
Plaque stress increased from 112.6 to $242.6 \mathrm{kPa}$. There was an increase of $100 \%$ in plaque stress when the lumen curvature was decreased by $38 \%$ (Fig 4).

An inverse relationship can be found between plaque stress and relative fibrous cap thickness (Fig 5A). Plaque stress increases exponentially when the fibrous cap thickness is decreased. When the fibrous cap is relatively thick $(d=0.3 \mathrm{D})$, there is only a $5 \%$ increase in predicted plaque stress, despite increasing the lipid core size by $500 \%$ (Fig 5B). This indicates that the size of the lipid core has no significant influence on plaque stress when the fibrous cap is relatively thick. When the fibrous cap is relatively thin $(\mathrm{d}=0.12 \mathrm{D})$, there is a large increase in plaque stress from when there is no lipid core to a small-sized lipid core $(A=0.4 D)$. After changing lumen shape from a circle to an ellipse by changing the ratio between 2 main axes (a and b), the predicted stress increased dramatically (Fig 5C). The 
A The effect of fibrous cap thickness on plaque stress

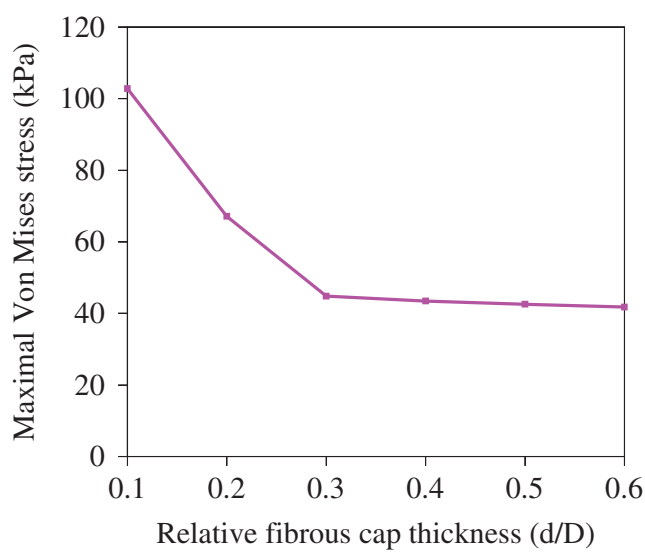

B The effect of lipid pool size on plaque stress

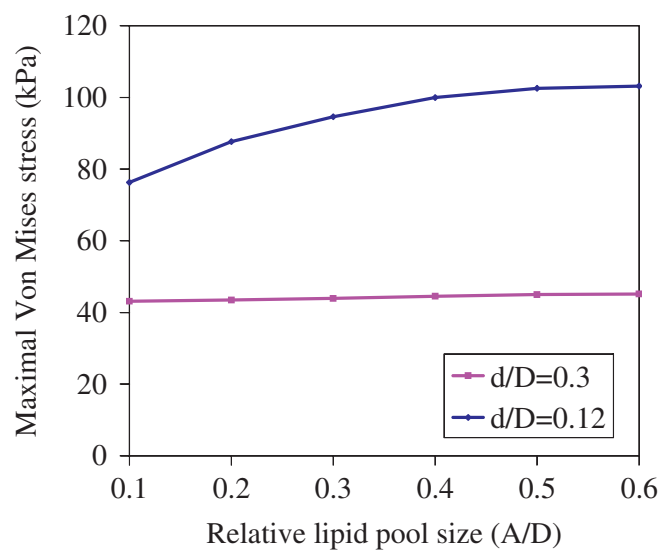

C The effect of lumen shape on plaque stress

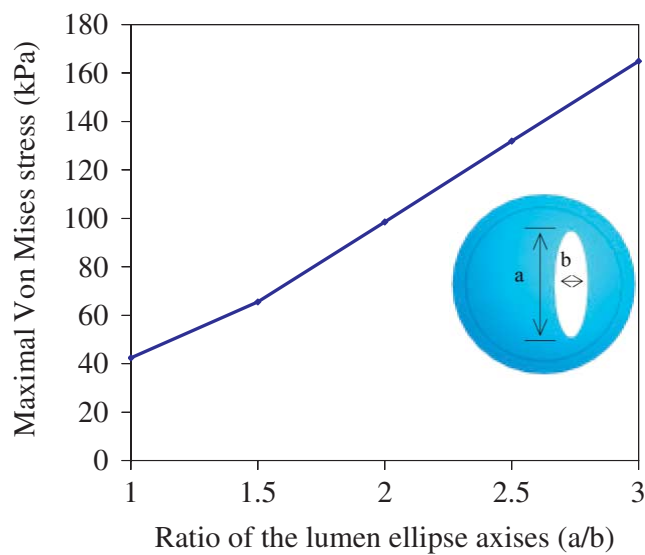

Fig 5. Maximal stresses within the plaque as a function of relative fibrous cap thickness (A), lipid core size (B), and lumen shape $(\mathrm{C})$.

Table 1 Baseline Characteristics of Symptomatic and Asymptomatic Patients

\begin{tabular}{lccc}
\hline & Symptomatic patients & Asymptomatic patients & $p$ value \\
\hline No. of patients & 20 & 20 & \\
Mean age, years (range) & $71.2(52-83)$ & $72.3(57-81)$ & 0.72 \\
Female (\%) & $5(25 \%)$ & $6(30 \%)$ & 0.99 \\
Hypertension (\%) & $15(75 \%)$ & $14(70 \%)$ & 0.99 \\
Diabetes $(\%)$ & $3(15 \%)$ & $2(10 \%)$ & 0.99 \\
PTCA/CABG (\%) & $2(10 \%)$ & $2(10 \%)$ & 1 \\
Peripheral vascular operations (\%) & $2(10 \%)$ & $4(20 \%)$ & 0.66 \\
Current smoker (\%) & $2(10 \%)$ & $3(15 \%)$ & 0.99 \\
Past smoker $(\%)$ & $13(65 \%)$ & $13(63 \%)$ & 1 \\
Never smoked $(\%)$ & $5(25 \%)$ & $4(20 \%)$ & 0.99 \\
Hypercholesterolemia $(\%)$ & $9(45 \%)$ & $10(50 \%)$ & 0.99 \\
Cholesterol mmol/L (SD) & $5.68( \pm 1.12)$ & $4.88( \pm 1.17)$ & 0.21 \\
Triglycerides mmol/L(SD) & $2.37( \pm 0.91)$ & $1.86( \pm 0.76)$ & 0.37 \\
Statin use $(\%)$ & $18(80 \%)$ & $20(100 \%)$ & 0.49 \\
Antiplatelet agent $(\%)$ & $18(80 \%)$ & $20(100 \%)$ & 0.49 \\
\hline
\end{tabular}

PTCA, percutaneous coronary angioplasty; CABG, coronary artery bypass graft.

stress concentrations can be found at both ends of the main axis.

Multi-sequence MRI studies were performed for all 40 patients. The baseline characteristics of the 2 groups are shown in Table 1. There was no significant difference in the severity of stenosis, as measured by carotid duplex, between the symptomatic (median $=71.5 \%$, range $52.5-87.0 \%$ ) and asymptomatic groups $($ median $=67.5 \%$, range $48.5-80.0 \%$ ) $(\mathrm{p}=0.36)$. Analysis revealed that all plaques had evidence of a lipid core and a fibrous cap. There were, however, very few foci of calcification.

An example of the structural analysis of a carotid atherosclerotic plaque based on plaque geometry derived from in vivo high-resolution MRI is illustrated in Fig 6. The axial 3-D TOF MR angiogram shows moderate carotid stenosis. Intermediate $\mathrm{T}_{2}$ weighted with fat saturation and STIR 


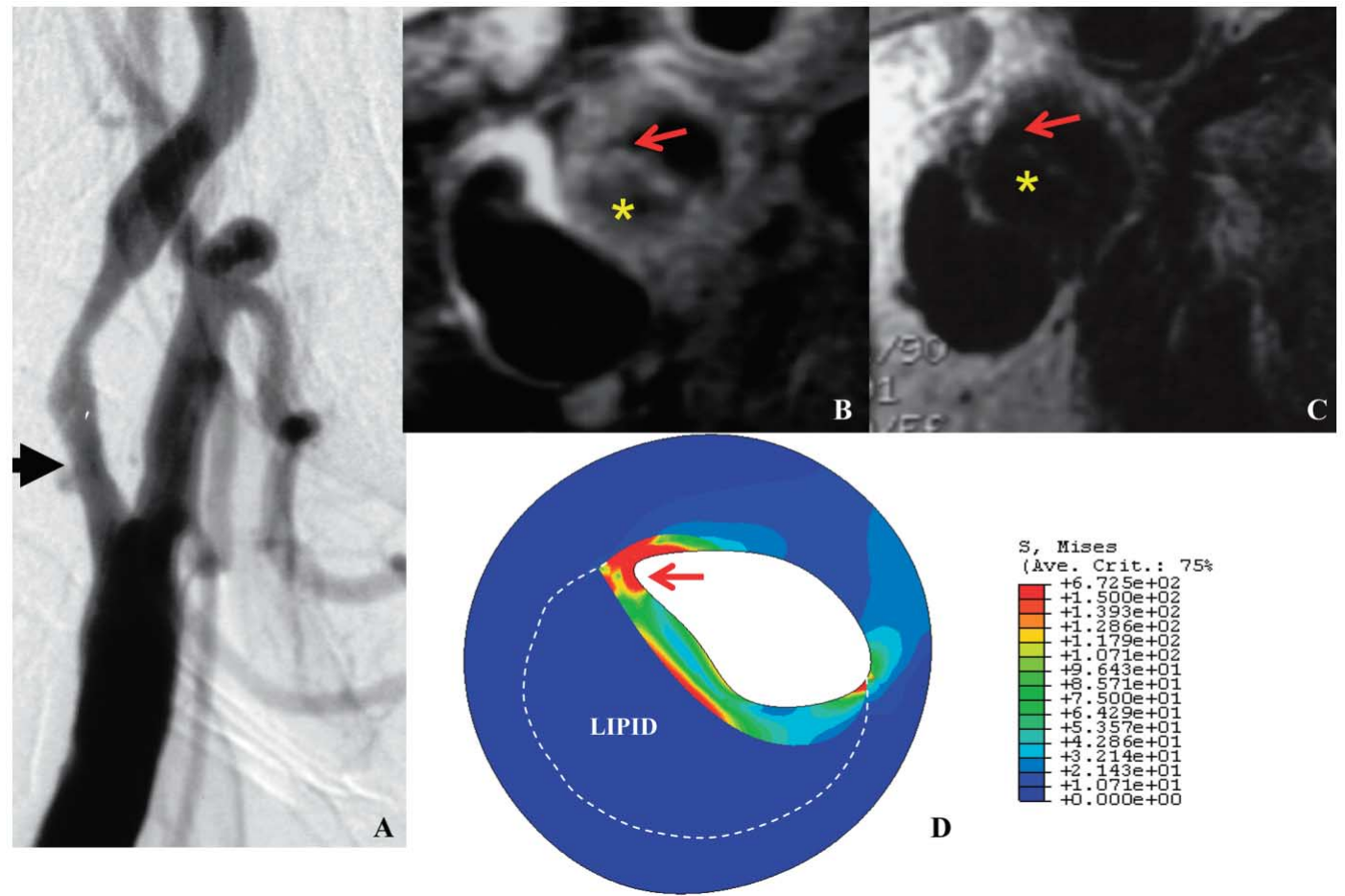

Fig 6. (A) Gated Fast Time-Of-Flight imaging of moderate carotid stenosis. (B, C) Intermediate $\mathrm{T} 2$ weighted with fat saturation and short $\mathrm{T}_{1}$ inversion-recovery depicting a complex plaque with thin fibrous cap (red arrow), overlying a large necrotic lipid core (*). (D) Von Mises stress map showing high stress $(672.5 \mathrm{kPa})$ (red arrow) at the shoulder region. This plaque would be at high risk of causing stroke and the patient would probably benefit from surgery.

imaging depicts a complex plaque with a thin fibrous cap, overlying a large necrotic lipid core. The stress map shows a high-stress concentration $(672.5 \mathrm{kPa})$ in the shoulder region of the plaque. The large stress concentration is thought to be caused by a combination of the very thin fibrous cap and large lumen curvature.

The 2 groups of data were tested for normality. The p-value suggested that the data were probably distributed normally and a normal plot superimposed on a frequency histogram further supported this hypothesis (symptomatic $\mathrm{W}=0.93, \mathrm{p}=0.35$; asymptomatic $\mathrm{W}=0.93$, $\mathrm{p}=0.47$ ). Following this evaluation of normality, parametric statistical analysis was undertaken using a non-paired T test (SPSS v12.0) and considered at the 5\% significance level.

The relationship between plaque maximum stress and plaque minimum lumen curvature was tested for both groups. The correlation between plaque stress and lumen curvature was found to be significant for both symptomatic ( $\mathrm{p}=0.01$; correlation coefficient: 0.689$)$ and asymptomatic groups ( $\mathrm{p}=0.01$; correlation coefficient: 0.862$)$. The maximum stresses in the plaques of the symptomatic patients were significantly higher than in those of asymptomatic patients $(519.2 \pm 109.9 \mathrm{kPa}$ vs $287.3 \pm 123.1 \mathrm{kPa}$ (95\% confidence interval $(\mathrm{CI})$ of the difference was 142.2 to $361.0 \mathrm{kPa}, \mathrm{p}=0.009)$ ). Lumen curvature of the plaques of symptomatic patients was significantly larger than those of asymptomatic patients $\left(1.50 \pm 1.0 \mathrm{~mm}^{-1}\right.$ vs $1.25 \pm 0.75 \mathrm{~mm}^{-1}$ (95\% CI of the difference was 0.5 to $\left.2.0 \mathrm{~mm}^{-1}, \mathrm{p}=0.01\right)$ ).

\section{Discussion}

We examined the effect of varying fibrous cap thickness, lipid core size and lumen curvature on plaque stress and our principal findings are as follows.

1. A thin fibrous cap leads to a high stress concentration.

2. Lipid core size does not alter plaque stress when the fibrous cap is thick.

3. Lumen shape and curvature play an important role in plaque stability. A large lumen curvature may result in a high stress concentration within the plaque.

4. Lumen curvature is positively correlated with plaque stress and was found to be significantly higher in symptomatic patients. Lumen curvature and structural analysis based on in vivo MRI may aid in identifying vulnerable patients.

A thin fibrous cap leads to a high stress concentration, which is consistent with previous in vivo and ex vivo studies! ${ }^{17} 19$ The thinner the fibrous cap, the greater the plaque stress. Plaque stress increases exponentially when the fibrous cap thickness is decreased. However, the size of the lipid core has no significant influence on plaque stress when the fibrous cap is relatively thick. These findings are consistent with those from a previous study of longitudinal plaque models $2^{3}$ Our results also show that there is a large increase in plaque stress from when there is no lipid core to a smallsized lipid core. This suggests that it is the existence of the lipid core, and not its size, that has the greatest impact on overall stress within plaques with a thin fibrous cap.

Increased plaque stress can often be found in the areas of increased lumen curvature, such as the plaque shoulder regions 17 The predicted stress increased dramatically when increasing the ratio between 2 main axes of the ellipse in the plaque model. The stress concentrations can be found at both ends of the main axis, which suggests that plaque with a large lumen curvature or sharp "corners" could be at high 
risk of rupture. Our study explains why stress concentrations are often be found in the shoulder regions of plaque, which often have a large lumen curvature at the junction of the fibrous cap and the vessel wall.

We found that plaque stress was significantly higher in symptomatic patients than in asymptomatic patients, as was found in a previous study ${ }^{29}$ Plaque stress positively correlated with lumen curvature in both symptomatic and asymptomatic patients and lumen curvature was significantly larger in symptomatic patients. These findings are consistent with each other and suggest that plaque stress or lumen curvature may be useful in the risk stratification of patients with carotid stenosis, especially for asymptomatic patients with moderate stenosis.

From a structural engineering point of view, risk assessment of plaque vulnerability needs quantification of plaque morphology. Luminal stenosis alone may not adequately reflect disease burden. For example, 1 patient with only $48 \%$ carotid stenosis had a maximal plaque stress of $464.12 \mathrm{kPa}$, whereas another patient with $80 \%$ carotid stenosis had a maximal plaque stress of only $203.94 \mathrm{kPa}$. This is because the plaque with less luminal stenosis had a vulnerable structure (thin fibrous cap and large lumen curvature) and the other with a higher degree of stenosis had a more stable structure (thick fibrous cap and round lumen). Furthermore, the measurement of luminal stenosis may underestimate the degree of plaque burden, because of vessel remodeling? 30 The advent of high-resolution imaging techniques, such as intravascular ultrasound (IVUS) and MRI, has enabled detailed morphological and structural characterization of carotid plaques to be performed in vivo, 6,31 Our analysis used geometric and morphological data derived from in vivo MRI of the carotid bifurcation in order to conduct FEA of plaque rupture. This is different from previous studies that used geometry derived from histology 22 or ex vivo MRI 32 This distinction is crucial because changes in the shape of vessels as a result of endarterectomy and histological fixation will have a significant impact on the predicted stress distributions $18,33,34$ The present study combined the advances in engineering techniques (eg, FEA) with in vivo highresolution multi-spectral MRI to examine the structural determinants of plaque stability, which may help in identifying vulnerable plaque in the future.

We did not study the effect of an applied load, a factor that is likely to be as important as morphological and biological information in determining stress distribution. An intra-luminal pressure of $115 \mathrm{mmHg}$ was used in all the simulations because it approximates the mean arterial pressure in humans; but as hypertension frequently accompanies atherosclerotic disease it is possible that in the target group with carotid disease, the applied load may be higher, despite concomitant antihypertensive medication.

It is true that actual lesions in humans are not always perfect circles, or even ellipses, and can have diverse shapes, and the pattern of lipid distribution within a plaque is also not predictable and uniform. This is why the actual stress in plaques based on in vivo MRI is higher than that based on idealized models. The simplified idealized models can be used to outline the major determinants for plaque stability, but in order to quantify the risk of plaque rupture for each individual patient, structural analysis based on in vivo MRI and quantification of the plaque components' materials properties needs to be conducted for each individual patient. Other limitation of this study is that plaque rupture was approached as a purely structural problem, rather than a biological one. Of course, plaque rupture is a dynamic biological, biochemical and biomechanical process, and only 1 aspect of this complex interaction was considered in our study. Biological and biochemical factors have received much attention, and this has led to a better understanding of tissue changes in diseased arteries and their correlation with plaque rupture. However, the concept of biomechanical rupture has been minimally studied and may provide insight into the complex mechanism of atheroma formation and rupture. Second, each component of the plaque was assumed to be isotropic and homogeneous. By assuming that the fibrous cap, lipid core, and normal arterial wall are characterized by a single set of structural parameters, spatial and inter-specimen variations within a particular component were not considered here. However, this assumption has been widely accepted for the assessment of the biomechanical properties of atherosclerotic lesions. ${ }^{17,19}$ There are only limited data available about the anisotropic properties of the biomechanical behavior of atherosclerotic lesions. Third, plaque calcification was not included in the model because there were very few foci of calcification seen during MRI. However, we have studied the impact of calcification on plaque stability based on MRI and histology 35 and found that the location rather than the size of the calcification may play an important role. Additional CT scanning could be used to detect plaque calcification and improve the accuracy of the modeling work. Finally, we assumed that there were no shear stresses, torques or time-dependent forces. Only static blood pressure was considered to be acting on the lesion in the models. It has been documented that the effect of fluid shear stress is insignificant when compared with the effect of tensile wall stresses ${ }^{21}$ as a direct component in plaque fracture dynamics. The estimation of stresses induced by static pressure load alone has already shown its usefulness in identifying stress concentration in human lesions, ${ }^{17}$ because the location of stress concentration does not significantly differ between the single static pressure model and the complex dynamic pressure model.

The approaches we used are very important for the management of patients with carotid stenosis because they help identify vulnerable plaque before it ruptures. The development of reliable methods for identifying vulnerable plaque, and intervention before rupture, will require improved understanding of the mechanism of plaque rupture. Although luminal stenosis is the clinical standard for diagnosing carotid artery disease, the method poorly predicts the site of subsequent plaque rupture that will lead to stroke. The present study indicates that characterization of the sub-intimal structure through imaging modalities such as MRI may be useful in identifying vulnerable plaque. Studies of idealized geometries suggest a thin fibrous cap and large lumen curvature may dramatically increase peak stress in the lesion. Our results obtained by comparing symptomatic and asymptomatic patients using the MRI-based model confirm that plaque stress may be an independent risk indicator for plaque stability. Therefore, quantification of plaque morphology and simulation of a plaque model based on in vivo MRI may improve the management of carotid patients in the future.

\section{Conclusion}

We used idealized and patient-specific models and examined the structural determinants of plaque stability. It has been validated that plaques with thin fibrous caps are at high 
risk of rupture. Large lumen curvature may result in high stress concentrations and explains why stress concentration can often be found at the shoulders of the plaque. Detailed plaque morphology quantification is needed for risk stratification of vulnerable plaque rather than by measurement of luminal stenosis or fibrous cap thickness alone. A combination of in vivo high-resolution MRI and structural analysis could prove to be a useful tool in the future for risk stratification of patients with carotid atheroma, particularly for asymptomatic patients with moderate luminal stenosis.

\section{Acknowledgments}

We gratefully acknowledge the support of The Stroke Association and GlaxoSmithKline. We thank Mr Tim Baynes, Specialist Research Nurse at Addenbrooke's Hospital, for his support.

\section{Reference}

1. Choy PC, Siow YL, Mymin D, O K. Lipids and atherosclerosis. Biochem Cell Biol 2004; 82: 212-224.

2. European Carotid Surgery Trialists' Collaborative Group. MRC European Carotid Surgery Trial: Interim results for symptomatic patients with severe (70-99\%) or with mild (0-29\%) carotid stenosis. Lancet 1991; 337: $1235-1243$

3. North American Symptomatic Carotid Endarterectomy Trial Collaborators. Beneficial effect of carotid endarterectomy in symptomatic patients with high-grade carotid stenosis. N Engl J Med 1991; 325: $445-453$

4. Halliday A, Mansfield A, Marro J, Peto C, Peto R, Potter J, et al. Prevention of disabling and fatal strokes by successful carotid endarterectomy in patients without recent neurological symptoms: Randomised controlled trial. Lancet 2004; 363: 1491-1502.

5. Hatsukami TS, Ross R, Polissar NL, Yuan C. Visualization of fibrous cap thickness and rupture in human atherosclerotic carotid plaque in vivo with high-resolution magnetic resonance imaging. Circulation 2000; 102: 959-964.

6. Lindstedt KA, Kovanen PT. Proteolysis of pericellular matrix: A process linking inflammation to plaque destabilization and rupture. Arterioscler Thromb Vasc Biol 2004; 24: 2205-2206.

7. Redgrave JN, Lovett JK, Gallagher PJ, Rothwell PM. Histological assessment of 526 symptomatic carotid plaques in relation to the nature and timing of ischemic symptoms: The Oxford plaque study. Circulation 2006; 113: 2320-2328.

8. Spagnoli LG, Mauriello A, Sangiorgi G, Fratoni S, Bonanno E, Schwartz RS, et al. Extracranial thrombotically active carotid plaque as a risk factor for ischemic stroke. JAMA 2004; 292: 1845-1852.

9. Takaya N, Yuan C, Chu B, Saam T, Underhill H, Cai J, et al. Association between carotid plaque characteristics and subsequent ischemic cerebrovascular events: A prospective assessment with MRI: Initial results. Stroke 2006; 37: 818-823.

10. Ouhlous M, Flach HZ, de Weert TT, Hendriks JM, van Sambeek MR, Dippel DW, et al. Carotid plaque composition and cerebral infarction: MR imaging study. Am J Neuroradiol 2005; 26: 1044-1049.

11. Kato M, Dote K, Sasaki S, Ueda K, Matsuda O, Nakano Y, et al. Coronary plaque vulnerability in metabolic syndrome: Assessment of carotid artery morphology in acute coronary syndrome. Circ J 2007; 71: $1229-1233$

12. Seo $\mathrm{Y}$, Watanabe $\mathrm{S}$, Ishizu T, Moriyama N, Takeyasu N, Maeda H, et al. Echolucent carotid plaques as a feature in patients with acute coronary syndrome. Circ J 2006; 70: 1629-1634.

13. Richardson PD. Biomechanics of plaque rupture: Progress, problems, and new frontiers. Ann Biomed Eng 2002; 30: 524-536.

14. Naghavi M, Libby P, Falk E, Casscells SW, Litovsky S, Rumberger J, et al. From vulnerable plaque to vulnerable patient: A call for new definitions and risk assessment strategies: Part II. Circulation 2003; 108: $1772-1778$

15. Naghavi M, Libby P, Falk E, Casscells SW, Litovsky S, Rumberger J, et al. From vulnerable plaque to vulnerable patient: A call for new definitions and risk assessment strategies: Part I. Circulation 2003; 108: $1664-1672$.

16. Rohde LE, Lee RT. Pathophysiology of atherosclerotic plaque development and rupture: An overview. Semin Vasc Med 2003; 3: $347-$ 354.

17. Cheng GC, Loree HM, Kamm RD, Fishbein MC, Lee RT. Distribution of circumferential stress in ruptured and stable atherosclerotic lesions: A structural analysis with histopathological correlation. Circulation 1993; 87: 1179-1187.

18. Lee RT. Atherosclerotic lesion mechanics versus biology. Z Kardiol 2000; 89(Suppl 2): 80-84.

19. Loree HM, Kamm RD, Stringfellow RG, Lee RT. Effects of fibrous cap thickness on peak circumferential stress in model atherosclerotic vessels. Circ Res 1992; 71: 850-858.

20. Lee RT, Loree HM, Cheng GC, Lieberman EH, Jaramillo N, Schoen FJ. Computational structural analysis based on intravascular ultrasound imaging before in vitro angioplasty: Prediction of plaque fracture locations. J Am Coll Cardiol 1993; 21: 777-782.

21. Huang H, Virmani R, Younis H, Burke AP, Kamm RD, Lee RT. The impact of calcification on the biomechanical stability of atherosclerotic plaques. Circulation 2001; 103: 1051-1056.

22. Tang D, Yang C, Zheng J, Woodard PK, Sicard GA, Saffitz JE, et al. 3D MRI-based multicomponent FSI models for atherosclerotic plaques. Ann Biomed Eng 2004; 32: 947-960.

23. Imoto K, Hiro T, Fujii T, Murashige A, Fukumoto Y, Hashimoto G, et al. Longitudinal structural determinants of atherosclerotic plaque vulnerability: A computational analysis of stress distribution using vessel models and three-dimensional intravascular ultrasound imaging. $J$ Am Coll Cardiol 2005; 46: 1507-1515.

24. Li ZY, Howarth SP, Tang T, Gillard JH. How critical is fibrous cap thickness to carotid plaque stability?: A flow-plaque interaction model. Stroke 2006; 37: 1195-1199.

25. Cai J, Hatsukami TS, Ferguson MS, Kerwin WS, Saam T, Chu B, et al. In vivo quantitative measurement of intact fibrous cap and lipidrich necrotic core size in atherosclerotic carotid plaque: Comparison of high-resolution, contrast-enhanced magnetic resonance imaging and histology. Circulation 2005; 112: 3437-3444.

26. Trivedi RA, U-King-Im J, Graves MJ, Horsley J, Goddard M, Kirkpatrick PJ, et al. Multi-sequence in vivo MRI can quantify fibrous cap and lipid core components in human carotid atherosclerotic plaques. Eur J Vasc Endovasc Surg 2004; 28: 207-213.

27. Li ZY, Howarth S, Trivedi RA, U-King-Im JM, Graves MJ, Brown $\mathrm{A}$, et al. Stress analysis of carotid plaque rupture based on in vivo high resolution MRI. J Biomech 2006; 39: 2611-2622.

28. Ogden RW. Large deformation isotropic elasticity: On the correlation of theory and experiment for incompressible rubberlike solids. Proc $R$ Soc Lond A 1972; 326: 565-584.

29. Li ZY, Howarth SP, Tang T, Graves MJ, U-King-Im J, Trivedi RA, et al. Structural analysis and magnetic resonance imaging predict plaque vulnerability: A study comparing symptomatic and asymptomatic individuals. J Vasc Surg 2007; 45: 768-775.

30. Pasterkamp G, Smits PC. Imaging of atherosclerosis: Remodelling of coronary arteries. J Cardiovasc Risk 2002; 9: 229-235.

31. Yuan C, Kerwin WS, Ferguson MS, Polissar N, Zhang S, Cai J, et al. Contrast-enhanced high resolution MRI for atherosclerotic carotid artery tissue characterization. J Magn Reson Imaging 2002; 15: $62-$ 67.

32. Morrisett J, Vick W, Sharma R, Lawrie G, Reardon M, Ezell E, et al. Discrimination of components in atherosclerotic plaques from human carotid endarterectomy specimens by magnetic resonance imaging ex vivo. Magn Reson Imaging 2003; 21: 465-474.

33. Rohde L, Lee RT. Mechanical stress and strain and the vulnerable atherosclerotic lesion. In: Fuster V (editor). The vulnerable atherosclerotic plaque: Understanding, identification and modification. New York: Futura Publishing Company; 1999; 305-316.

34. Loree HM, Tobias BJ, Gibson LJ, Kamm RD, Small DM, Lee RT. Mechanical properties of model atherosclerotic lesion lipid pools. Arterioscler Thromb 1994; 14: 230-234.

35. Li ZY, Howarth S, Tang T, Graves M, U-King-Im J, Gillard JH. Does calcium deposition play a role in the stability of atheroma?: Location may be the key. Cerebrovasc Dis 2007; 24: 452-459. 